\title{
Atherosclerosis and interferon-y: New insights and therapeutic targets
}

\author{
Iryna Voloshyna, Michael J. Littlefield, and Allison B. Reiss * \\ Department of Medicine, Winthrop Research Institute, Winthrop-University Hospital, 222 Station \\ Plaza North, Mineola, NY 11501
}

\section{Abstract}

Atherosclerosis is considered to be a chronic inflammatory disease of the arterial wall. Atherogenesis is accompanied by local production and release of inflammatory mediators, for which the macrophage is a major source. The proinflammatory cytokine, interferon (IFN)- $\gamma$ derived from $\mathrm{T}$ cells, is expressed at high levels in atherosclerotic lesions. IFN- $\gamma$ is the classic macrophage-activating factor, vital for both innate and adaptive immunity. It primes macrophages to produce chemokines and cytotoxic molecules and induces expression of genes that regulate lipid uptake. IFN- $\gamma$ is a key trigger for the formation and release of reactive oxygen species. IFN- $\gamma$ has important effects on endothelial cells, promoting expression of adhesion molecules. Atherogenic effects of IFN- $\gamma$ have been shown in murine models where exogenous administration enhances atherosclerotic lesion formation while knockout of IFN- $\gamma$ or its receptor reduces lesion size. IFN- $\gamma$ signaling is largely mediated by a Janus kinase (JAK) to signal transduction and activator of transcription (STAT) 1 cytosolic factor pathway. A clear understanding of IFN- $\gamma$ effects on atherogenesis should enable development of novel targeted interventions for clinical use in the prevention and treatment of atherosclerosis. This review will discuss the actions of the cytokine IFN- $\gamma$ and its complex effects on cells involved in atherosclerosis.

\section{Introduction}

Atherosclerosis involves an ongoing inflammatory response (Libby, 2012). The atherosclerotic plaque consists of large amounts of inflammatory cells, mainly monocytes/ macrophages and $\mathrm{T}$ lymphocytes. Inflammatory markers are found inside affected vessel walls and in the plasma of patients with atherosclerotic vascular disease (Lind, 2003). Unraveling the details of inflammatory pathways in the vessel wall has direct application in the detection and prevention of cardiovascular disease, and current research is predominantly focused on understanding the impact of this inflammation and how it might be controlled. The pathogenesis of atherosclerosis involves the interplay between cholesterol and cellular secretion of cytokines, a major one being the pleiotropic soluble cytokine interferon- $\gamma$ (IFN- $\gamma)$, which is secreted by T lymphocytes and macrophages. IFN- $\gamma$ is involved in the initiation and modulation of a variety of immune responses, many of which are pro-atherogenic (Billiau and Matthys, 2009). IFN- $\gamma$ is vital for both innate and adaptive immunity and exerts its effects by activating macrophages, natural killer cells, and B cells. Stimulation of endothelial cells by IFN- $\gamma$ promotes expression of adhesion molecules. Macrophage activation by IFN- $\gamma$ leads to the production of other proinflammatory cytokines like tumor necrosis factor (TNF)- $a$ and interleukin (IL)-6, oxygen radicals, and 
metalloproteinases. Both protein and mRNA for IFN- $\gamma$ have been detected in human atherosclerotic lesions where it colocalizes with activated major histocompatibility complex (MHC)-II-expressing macrophages (Tedgui and Mallat, 2006). This review will examine the impact of IFN- $\gamma$ on the cell types within the vessel wall that participate in the atherosclerotic process and the interconnected cascade of events that ultimately form atherosclerotic plaque that is vulnerable to rupture.

\section{IFN-y signaling}

IFNs mediate their effects through activation of intracellular molecular signaling networks, the best characterized of which is the Janus kinase-signal transducer and activator of transcription (JAK)-STAT pathway (Tedgui and Mallat, 2006) (Fig. 1). The IFN- $\gamma$ receptor is composed of two subunits (IFNGR1 and IFNGR2) that dimerize upon ligand binding leading to transactivation of JAK1 and 2. The activation of JAKs upon cytokine stimulation results in the phosphorylation of the intracellular domain of the receptor subunit IFNGR1, forming a docking site for STAT1, which is phosphorylated at Tyr ${ }^{701}$. Phospho-STAT1 dimerizes, dissociates from the receptor cytoplasmic domain, and translocates to the nucleus to activate gene transcription (Varinou et al., 2003). During early activation, an independent phosphorylation takes place at the $\operatorname{Ser}^{727}$ motif of STAT1. Ser ${ }^{727}$ phosphorylation is required for the majority of IFN- $\gamma$-induced transcriptional activity but does not mediate DNA binding or nuclear translocation (Li et al., 2010; Fig. 1).

Li et al. (2010) demonstrated an important role of extracellular signal-regulated kinase (ERK), JNK, protein kinase C (PKC) $\delta$, and calcium calmodulin-dependent kinase (CamK) II in the Ser ${ }^{727}$ phosphorylation of STAT1 in the THP-1 human macrophage cell line. Inhibition or knockdown of ERK attenuated IFN- $\gamma$-mediated uptake of modified low-density lipoprotein (LDL) and release of chemokines and adhesion molecules.

\section{The role of IFN- $\mathrm{y}$ in atherosclerosis}

IFN- $\gamma$ affects cholesterol accumulation in monocytes/macrophages, induces foam cell formation, and participates in adaptive T-helper type 1 (Th1)-specific immune response, atherosclerotic plaque formation, and rupture. $\mathrm{CD}^{+} \mathrm{T}$ lymphocytes in atherosclerotic lesions are predominately Th1 cells. Through IFN- $\gamma$ production, Th1-type cells stimulate macrophages leading to production of proinflammatory cytokines and vasoactive molecules. Th1 bias drives increased atherosclerosis in mice and recent evidence supports a similar effect of Th1 bias in humans (Tracy et al., 2013). IFN- $\gamma$ administration also significantly increases T-cell number within lesions in atherosclerosis-prone apolipoprotein (Apo) E knockout mice (Whitman et al., 2000).

Numerous lines of evidence signify a role for IFN- $\gamma$ in induction, development, and progression of atherosclerosis. These steps will be summarized in the sections that follow.

\section{IFNy, oxidative stress, and initiation of atherosclerosis}

LDL possesses the ability to pass from the blood into and out of the intimal layer of the vessel. LDL accumulates in the intima and binds to matrix glycoproteins, forming lipid droplets and fatty streaks. Lipoproteins are subjected to chemical modifications, such as oxidation, aggregation, or glycation. Superoxide or superoxide-derived reactive oxygen species (ROS) are chemically reactive biomolecules that promote LDL oxidation (Heistad et al., 2009). Induced by ROS, oxidatively modified LDL is recognizable to macrophage scavenger receptors, resulting in lipid uptake and foam cell transformation (Steinberg, 2002). Therefore, the superoxide-producing enzyme NADPH oxidase, considered to be the most important inducer of ROS production, has been strongly linked to atherosclerosis. IFN- 
$\gamma$ is able to upregulate the expression of NADPH oxidase in human monocyte-derived macrophages.

Concurrently with oxidative-stress induction, stimulation of human macrophages by IFN- $\gamma$ increases concentrations of the immune activation marker-neopterin. Neopterin may act pro-oxidatively and has been found to support LDL oxidation in vitro (Schroecksnadel et al., 2006; Fig. 2). High concentration of neopterin is strongly associated with the extent of atherosclerosis (Sugioka et al., 2010).

Cells have developed several antioxidant mechanisms to control oxidizing activity. However, with increased production of ROS, these antioxidant systems are overloaded and fail to respond properly. Accumulation of ROS leads to immune system activation (recruitment of monocytes and $\mathrm{T}$ lymphocytes) and development of oxidative stress. In a prospective study of patients with stable documented coronary artery disease, oxidative stress, as indicated by a heightened blood flow response to vitamin C infusion, was found to contribute significantly to atherosclerosis progression and outcome in cardiovascular disease (Heitzer et al., 2001).

The ability of IFN- $\gamma$ to upregulate inducible nitric oxide synthases (iNOS) gene expression in macrophages (Chung et al., 2011) can be considered an anti-atherogenic effect, provided the nitric oxide level does not rise excessively. Local release of a large amount of nitric oxide metabolites has been linked to vascular hyperreactivity and the production of harmful oxidative products such as peroxynitrite. Moreover, neopterin also accelerates oxidation of nitric oxide to form peroxynitrite. Peroxynitrite may enhance platelet adhesion and aggregation, promotes lipid peroxidation, increases vasoconstriction, and raises blood pressure (Dusting et al., 2005).

\section{IFN-y and activation of the arterial endothelium}

The damage of the endothelial lining of the arterial wall, intervened by oxidative stress, is considered as an initial step of atherosclerosis. Activated endothelium expresses monocyte chemotactic protein 1 (MCP-1), macrophage colony-stimulating factor (M-CSF), and adhesion molecules, such as intercellular adhesion molecule 1 (ICAM-1), vascular cell adhesion molecule 1 (VCAM-1), E-selectin, and P-selectin. These molecules induce monocyte/lymphocyte recruitment and infiltration into the subendothelium.

In their resting state, endothelial cells interact poorly with leukocytes. IFN- $\gamma$ potentiates VCAM-1 expression induced by proinflammatory cytokines, such as TNF-a in endothelial cells, supporting leukocyte adhesion and recruitment (Zhang et al., 2011). IFN- $\gamma$ amplification of the TNF-a effect on VCAM-1 is regulated at the transcriptional level and depends on the nuclear transcription factor interferon-related factor (IRF)-1 (Lechleitner et al., 1998; Fig. 3).

Stimulated by IFN- $\gamma$, macrophages in co-culture with human umbilical vein endothelial cells activate nuclear factor-kappa B (NF- $\mathrm{kb}$ ) and induce MCP-1 gene expression in the endothelial cells (Rimbach et al., 2000). IFN- $\gamma$ plays a key role in the endothelium-mediated recruitment of monocytes and T lymphocytes in atherosclerotic lesions. In wild type C57BL/ 6 mice, T-cell adhesion to endothelial cells is induced by hypercholesterolemia, but this effect is diminished in IFN- $\gamma$ knockout mice (Stokes et al., 2003). In ApoE knockout mice, IFN- $\gamma$ deficiency reduced levels of macrophages and T lymphocytes in atherosclerotic lesions compared to single ApoE knockout mice not deficient in IFN- $\gamma$. However, these effects were restricted to male mice (Whitman et al., 2002). 
IFN- $\gamma$ can also act to inhibit endothelial cell proliferation via the human interferon-inducible protein 10 (IP-10), also known as chemokine ligand (CXCL) 10, a chemokine inducible by IFN- $\gamma$. A clear association has been found between increased serum levels of IP-10 and risk of coronary heart disease (Rothenbacher et al., 2006).

Exposure of endothelial cells to the combination of TNF-a and IFN- $\gamma$ results in the production of F11R, a member of the immunoglobulin superfamily of cell adhesion molecules (Azari et al., 2011). The F11R inserts into the luminal surface of the endothelium where it causes the surface to become thrombogenic, resulting in adhesion of platelets (Fig. $3)$.

\section{IFN-y and foam cell formation}

During atherogenesis, blood monocytes roll and adhere to the endothelium, transmigrate into the subendothelial space, and differentiate into macrophages. These macrophages ingest trapped modified LDL and become cholesterol-rich foam cells forming pathological intima thickening (Moore and Tabas, 2011). Lipid overload in macrophages occurs when the balance between cellular cholesterol inflow and outflow is disrupted. Cholesterol efflux from cells of the arterial wall to extracellular acceptors involves the ATP-binding cassette transporters (ABC) A1 and G1 (Voloshyna and Reiss, 2011). Synthesis of 27hydroxycholesterol by the mitochondrial cytochrome P450 27-hydroxylase (CYP27A1) in arterial endothelium and macrophages provides a pathway for elimination of intracellular cholesterol. 27-Hydroxycholesterol has statin-like properties, suppressing smooth muscle cell (SMC) proliferation and diminishing macrophage foam cell formation (Reiss et al., 2001). Proteins involved in lipid uptake into cells, particularly modified LDL, include the scavenger receptor CD36, scavenger receptor (SR)-AI, and lectin-like oxidized low-density lipoprotein receptor (LOX)-1 (Kzhyshkowska et al., 2012). Their expression is not diminished upon exposure to excess cholesterol, leading to foam cell formation and early atherosclerotic lesions. Another scavenger receptor, CXCL16, which functions as both a chemokine and a scavenger receptor, is expressed on the surface of human macrophages as well as aortic SMC and has been linked to atherogenesis (Wuttge et al., 2004).

Multiple lines of evidence indicate that the pro-atherogenic effects of IFN- $\gamma$ in human macrophages occur via the JAK/STAT signaling pathway. IFN- $\gamma$ signaling mediates macrophage lipid metabolism resulting in upregulation of scavenger receptors expression and impeding reverse cholesterol transport thus promoting lipid overload and foam cell formation (Table 1).

Macrophage-derived foam cells that accumulate in the intima produce a variety of secreted factors, such as platelet-derived growth factor (PDGF) and matrix metalloproteinases (MMPs) that contribute to further development of the atherosclerotic lesion. These factors stimulate recruitment and proliferation of SMC in the intima from the medial layer. IFN- $\gamma$ induced release of MMPs enables SMC to migrate through tissue by degrading extracellular matrix (Owens et al., 2004). Mature SMC retain remarkable plasticity and can become macrophage-like: accumulate cholesterol and contribute to the foam cell population of the intima (Rong et al., 2003).

\section{IFN-y and plaque development}

Early-stage fatty lesions are predominantly composed of lipid-enriched macrophages. The more advanced, complex lesion develops upon arrival of SMC from the arterial media, and their contribution to the foam cell population. Recruited SMC undergo a change from a contractile phenotype to a matrix synthetic phenotype (Rekhter et al., 1993). These SMC release collagen type I-the most abundant fibrillar protein in atherosclerotic lesions. In 
atherosclerotic arteries, collagen confers tensile strength and is crucial for plaque stability. SMC proliferation and the expression of collagen genes (collagens I and III) are inhibited by IFN- $\gamma$ (Amento et al., 1991).

Further progression of atherosclerosis involves influx of additional monocytes, arrival of $\mathrm{T}$ cells, and migration and proliferation of SMC. However, macrophage and SMC death by apoptosis may also occur. Ultimately, a fibrous cap forms, consisting of SMC and extracellular matrix, surrounding a necrotic core of debris that results from the death of accumulated foam cells by apoptosis or necrosis. Apoptosis is minimal in early lesions and peaks in advanced plaques (Clarke et al., 2006). Apoptosed macrophage-derived foam cells are not efficiently removed from advanced atherosclerotic lesions and appear to contribute to the accumulation of the necrotic lipid core. Several studies indicate that the balance between pro- and anti-inflammatory cytokines is a major determinant of plaque stability. Nonetheless, many aspects of plaque formation and evolution remain unresolved.

Munn et al. (1995) demonstrated that IFN- $\gamma$ can trigger apoptosis in human monocytes/ macrophages while Inagaki et al. (2002) documented apoptotic cell death induced by IFN- $\gamma$ in the THP-1 human macrophage cell line. Schaub et al. (2000) described a similar effect of IFN- $\gamma$ in vascular SMC. Inagaki et al. (2002) found that THP-1 macrophages stimulated by IFN- $\gamma$ to begin apoptosis also generated MCP-1 and this effect, if reproducible in vivo, might attract more monocytes to the intima. IFN- $\gamma$-induced release of MMPs in macrophages and vascular cells could also promote cell apoptosis, resulting in weakened plaques that are more prone to rupture or erosion. The plaque rupture is linked to the clinical complications of atherosclerosis, including myocardial infarction and stroke.

\section{New therapeutic targets}

Recent findings point to an important immunosuppressive role for the endogenous autacoid adenosine in macrophage-mediated inflammation. Macrophages have been reported to express all four subtypes of the adenosine receptor: A1, A2A, A2B, and A3. Ligation of one or more of these receptors suppresses the production of proinflammatory factors, stimulates expression of reverse cholesterol transport proteins, and inhibits macrophage foam cell formation.

Lee et al. (2011) demonstrated that adenosine A3 receptor stimulation reduces IFN- $\gamma$ induced STAT1 transcriptional activity and STAT1-dependent gene expression. Barnholt et al. (2009) revealed that in human macrophages adenosine affects IFN- $\gamma$ action by blocking of $\operatorname{Ser}^{727}$ STAT1 phosphorylation.

Resveratrol (3,5,4'-trihydroxy-trans-stilbene), a plant-derived dietary bioactive agent found in high concentrations in red wine and grape skin, exerts multiple beneficial effects on health due to its anti-inflammatory, cardioprotective, and cancer chemopreventive properties. Our group (Voloshyna et al., 2013) demonstrated that resveratrol mediates antiatherogenic effects on cholesterol flux in human macrophages and endothelium via PPAR- $\gamma$ and adenosine A2A receptor pathways. In murine RAW 264.7 macrophages, treatment with resveratrol reduced nitric oxide production induced by lipopolysaccharide alone or in combination with IFN- $\gamma$ (Chung et al., 2011) and inhibited IP-10 production. Moreover, resveratrol inhibited IFN- $\gamma$-induced activation of JAK2 and $\mathrm{Tyr}^{701}$ or Ser ${ }^{727}$ phosphorylation of STAT1.

MicroRNAs (miRs), short, endogenous, noncoding, single-stranded RNAs, have recently been recognized as gene regulators that work by promoting RNA instability. One particular $\mathrm{miR}, \mathrm{miR}-155$, is upregulated in murine atherosclerosis models and in human plaques, specifically in macrophages and some SMC (Nazari-Jahantigh et al., 2012). Murine bone 
marrow-derived macrophages treated with mildly oxidized LDL and IFN- $\gamma$ exhibited polarization to a proinflammatory M1 phenotype with increased expression of miR-155. Effects of miR-155 may be pro-atherogenic via repression of B-cell lymphoma 6, a transcription factor that attenuates proinflammatory NF- $\mathrm{kB}$ signaling. Atherogenicity of miR-155 is controversial and may be situation-specific. In oxidized-LDL-stimulated THP-1 macrophages, the miR-155 sequence reduces lipid uptake (Huang et al., 2010). Exploring IFN- $\gamma$ effects on miRs may open up new avenues of approach to therapy for atherosclerosis.

\section{Conclusions}

IFN- $\gamma$ is a Th 1 cytokine with well-documented atheroma-promoting properties. IFN- $\gamma$ has multiple effects on all stages of atherogenesis, and there has been progress in understanding the impact of this cytokine on signaling pathways that ultimately lead to plaque development and maturation. In early phases of atherosclerosis, IFN- $\gamma$ fosters adhesion molecule release from endothelial cells and is a crucial regulator of SMC proliferation. In later stages, IFN- $\gamma$ can create plaque vulnerability via accelerated macrophage apoptosis and extracellular matrix breakdown. Wielding this knowledge to develop innovative cardiovascular treatments is gaining attention. There has been some interest in targeting STAT1 using small molecules to block its action. Adenosine ligation, resveratrol, and related stilbenes also show promise. As further investigation deepens our knowledge of IFN- $\gamma$-dependent processes in atherosclerosis, there will likely be exciting opportunities to exploit IFN- $\gamma$ as a molecular therapeutic target for prevention and treatment of cardiovascular disease.

\section{Acknowledgments}

This work was supported by the Elizabeth Daniell Research Fund and by NIH/NCCAM Grant R21AT007032-01A1.

\section{REFERENCES}

Amento EP, Ehsani N, Palmer H, Libby P. Cytokines and growth factors positively and negatively regulate interstitial collagen gene expression in human vascular smooth muscle cells. Arteriosclerosis Thrombosis and Vascular Biology. 1991; 11:1223-30.

Azari BM, Marmur JD, Salifu MO, Ehrlich YH, Kornecki E, Babinska A. Transcription and translation of human F11R gene are required for an initial step of atherogenesis induced by inflammatory cytokines. Journal of Translational Medicine. 2011; 9:98. [PubMed: 21703019]

Barnholt KE, Kota RS, Aung HH, Rutledge JC. Adenosine blocks IFN-gamma-induced phosphorylation of STAT1 on serine 727 to reduce macrophage activation. Journal of Immunology. 2009; 183(10):6767-77.

Billiau A, Matthys P. Interferon-gamma: a historical perspective. Cytokine and Growth Factor Reviews. 2009; 20(2):97-113. [PubMed: 19268625]

Businaro R, Tagliani A, Buttari B, Profumo E, Ippoliti F, Di Cristofano C, et al. Cellular and molecular players in the atherosclerotic plaque progression. Annals of the New York Academy of Sciences. 2012; 1262:134-41. [PubMed: 22823445]

Clarke MC, Figg N, Maguire JJ, Davenport AP, Goddard M, Little-wood TD, et al. Apoptosis of vascular smooth muscle cells induces features of plaque vulnerability in atherosclerosis. Nature Medicine. 2006; 12:1075-80.

Chung EY, Kim BH, Hong JT, Lee CK, Ahn B, Nam SY, et al. Resveratrol down-regulates interferon$\gamma$-inducible inflammatory genes in macrophages: molecular mechanism via decreased STAT-1 activation. Journal of Nutritional Biochemistry. 2011; 22(10):902-9. [PubMed: 21189227]

Dusting GJ, Selemidis S, Jiang F. Mechanisms for suppressing NADPH oxidase in the vascular wall. Memorias do Instituto Oswaldo Cruz. 2005; 100(suppl 1):97-103. [PubMed: 15962105] 
Grewal T, Priceputu E, Davignon J, Bernier L. Identification of a gamma-interferon-responsive element in the promoter of the human macrophage scavenger receptor A gene. Arteriosclerosis Thrombosis and Vascular Biology. 2001; 21(5):825-31.

Hao XR, Cao DL, Hu YW, Li XX, Liu XH, Xiao J, et al. IFN-gamma down-regulates ABCA1 expression by inhibiting LXRalpha in a JAK/STAT signaling pathway-dependent manner. Atherosclerosis. 2009; 203(2):417-28. [PubMed: 18789440]

Heistad DD, Wakisaka Y, Miller J, Chu Y, Pena-Silva R. Novel aspects of oxidative stress in cardiovascular diseases. Circulation Journal. 2009; 73:201-7. [PubMed: 19110503]

Heitzer T, Schlinzig T, Krohn K, Meinertz T, Munzel T. Endothelial dysfunction, oxidative stress, and risk of cardiovascular events in patients with coronary artery disease. Circulation. 2001; 104:2673-8. [PubMed: 11723017]

Huang RS, Hu GQ, Lin B, Lin ZY, Sun CC. MicroRNA-155 silencing enhances inflammatory response and lipid uptake in oxidized low-density lipoprotein-stimulated human THP-1 macrophages. Journal of Investigative Medicine. 2010; 58:961-7. [PubMed: 21030878]

Inagaki Y, Yamagishi S, Amano S, Okamoto T, Koga K, Makita Z. Interferon-gamma-induced apoptosis and activation of THP-1 macrophages. Life Sciences. 2002; 71(21):2499-508. [PubMed: 12270755]

Kzhyshkowska J, Neyen C, Gordon S. Role of macrophage scavenger receptors in atherosclerosis. Immunobiology. 2012; 217(5):492-502. [PubMed: 22437077]

Lechleitner B, Gille J, Johnson DR, Petzelbauer P. Interferon enhances tumor necrosis factor-induced vascular cell adhesion molecule 1 (CD106) expression in human endothelial cells by an interferon related factor 1-dependent pathway. Journal of Experimental Medicine. 1998; 187:2023-30. [PubMed: 9625761]

Lee HS, Chung HJ, Lee HW, Jeong LS, Lee SK. Suppression of inflammation response by a novel $\mathrm{A}_{3}$ adenosine receptor agonist thio-Cl-IB-MECA through inhibition of Akt and NF- $\kappa \mathrm{B}$ signaling. Immunobiology. 2011; 216(9):997-1003. [PubMed: 21514967]

Li N, McLaren JE, Michael DR, Clement M, Fielding CA, Ramji DP. ERK is integral to the interferon-gamma-mediated activation of STAT1, the expression of key genes implicated in atherosclerosis, and the uptake of modified lipoproteins by human macrophages. Journal of Immunology. 2010; 185:3041-8.

Libby P. Inflammation in atherosclerosis. Arteriosclerosis Thrombosis and Vascular Biology. 2012; 32(9):2045-51.

Lind L. Circulating markers of inflammation and atherosclerosis. Atherosclerosis. 2003; 169:203-14. [PubMed: 12921971]

McLaren JE, Ramji DP. Interferon gamma: a master regulator of atheroslerosis. Cytokine and Growth Factor Reviews. 2009; 20:125-35. [PubMed: 19041276]

Moore KJ, Tabas I. Macrophages in the pathogenesis of atherosclerosis. Cell. 2011; 145:341-55. [PubMed: 21529710]

Munn DH, Beall AC, Song D, Wrenn RW, Throckmorton DC. Activation-induced apoptosis in human macrophages: developmental regulation of a novel cell death pathway by macrophage colonystimulating factor and interferon gamma. Journal of Experimental Medicine. 1995; 181(1):127-36. [PubMed: 7806999]

Nazari-Jahantigh M, Wei Y, Noels H, Akhtar S, Zhou Z, Koenen RR, et al. MicroRNA-155 promotes atherosclerosis by repressing Bcl6 in macrophages. Journal of Clinical Investigation. 2012; 122:4190-202. [PubMed: 23041630]

Owens GK, Kumar MS, Wamhoff BR. Molecular regulation of vascular smooth muscle cell differentiation in development and disease. Physiological Reviews. 2004; 84:767-801. [PubMed: 15269336]

Paulnock DM, Demick KP, Coller SP. Analysis of interferon-gamma-dependent and -independent pathways of macrophage activation. Journal of Leukocyte Biology. 2000; 67(5):677-82. [PubMed: 10811008]

Reiss AB, Awadallah NW, Malhotra S, Montesinos MC, Chan ES, Javitt NB. Immune complexes and IFN-gamma decrease cholesterol 27-hydroxylase in human arterial endothelium and macrophages. Journal of Lipid Research. 2001; 42(11):1913-22. [PubMed: 11714861] 
Reiss AB, Patel CA, Rahman MM, Chan ES, Hasneen K, Montesinos MC, et al. Interferon- $\gamma$ impedes reverse cholesterol transport and promotes foam cell transformation in THP-1 human monocytes/ macrophages. Medical Science Monitor. 2004; 10:BR420-5. [PubMed: 15507847]

Rekhter MD, Zhang K, Narayanan AS, Phan S, Schork MA, Gordon D. Type I collagen gene expression in human atherosclerosis. Localization to specific plaque regions. American Journal of Pathology. 1993; 143:1634-48. [PubMed: 7504887]

Rimbach G, Valacchi G, Canali R, Virgili F. Macrophages stimulated with IFN-gamma activate NFkappa B and induce MCP-1 gene expression in primary human endothelial cells. Molecular Cell Biology Research Communications. 2000; 3(4):238-42. [PubMed: 10891398]

Rong JX, Shapiro M, Trogan E, Fisher EA. Transdifferentiation of mouse aortic smooth muscle cells to a macrophage-like state after cholesterol loading. Proceedings of the National Academy of Sciences of the United States of America. 2003; 100(23):13531-6. [PubMed: 14581613]

Rothenbacher D, Müller-Scholze S, Herder C, Koenig W, Kolb H. Differential expression of chemokines, risk of stable coronary heart disease and correlation with established cardiovascular risk markers. Arteriosclerosis Thrombosis and Vascular Biology. 2006; 26:194-9.

Schaub FJ, Han DK, Liles WC, Adams LD, Coats SA, Ramachandran RK, et al. Fas/FADD-mediated activation of a specific program of inflammatory gene expression in vascular smooth muscle cells. Nature Medicine. 2000; 6(7):790-6.

Schrijvers DM, De Meyer GR, Kockx MM, Herman AG, Martinet W. Phagocytosis of apoptotic cells by macrophages is impaired in atherosclerosis. Arteriosclerosis Thrombosis and Vascular Biology. 2005; 25(6):1256-61.

Schroecksnadel K, Frick B, Winkler C, Fuchs D. Crucial role of interferon-gamma and stimulated macrophages in cardiovascular disease. Current Vascular Pharmacology. 2006; 4:205-13. [PubMed: 16842138]

Steinberg D. Atherogenesis in perspective: hypercholesterolemia and inflammation as partners in crime. Nature Medicine. 2002; 8:1211-7.

Stokes KY, Clanton EC, Clements KP, Granger DN. Role of interferon-gamma in hypercholesterolemia-induced leukocyte-endothelial cell adhesion. Circulation. 2003; 107:2140-5. [PubMed: 12695304]

Sugioka K, Naruko T, Matsumura Y, Shirai N, Hozumi T, Yoshiyama M, et al. Neopterin and atherosclerotic plaque instability in coronary and carotid arteries. Journal of Atherosclerosis and Thrombosis. 2010; 17(11):1115-21. [PubMed: 20693747]

Tedgui A, Mallat Z. Cytokines in atherosclerosis: pathogenic and regulatory pathways. Physiological Reviews. 2006; 86:515-81. [PubMed: 16601268]

Tenger C, Sundborger A, Jawien J, Zhou X. IL-18 accelerates atherosclerosis accompanied by elevation of IFN-gamma and CXCL16 expression independently of T cells. Arteriosclerosis Thrombosis and Vascular Biology. 2005; 25(4):791-6.

Tracy RP, Doyle MF, Olson NC, Huber SA, Jenny NS, Sallam R, et al. T-helper type 1 bias in healthy people is associated with cytomegalovirus serology and atherosclerosis: the multiethnic study of atherosclerosis. Journal of the American Heart Association. 2013; 202:e000117. [PubMed: 23688675]

Varinou L, Ramsauer K, Karaghiosoff M, Kolbe T, Pfeffer K, Muller M, et al. Phosphorylation of the Stat 1 transactivation domain is required for full-fledged IFN-gamma-dependent innate immunity. Immunity. 2003; 19:793-802. [PubMed: 14670297]

Voloshyna I, Reiss AB. The ABC transporters in lipid flux and atherosclerosis. Progress in Lipid Research. 2011; 50(3):213-24. [PubMed: 21352852]

Voloshyna I, Hai O, Littlefield MJ, Carsons SE, Reiss AB. Resveratrol mediates anti-atherogenic effects on cholesterol flux in human macrophages and endothelium via PPAR- $\gamma$ and adenosine. European Journal of Pharmacology. 2013; 698(1-3):299-309. [PubMed: 23041272]

Wang XQ, Panousis CG, Alfaro ML, Evans GF, Zuckerman SH. Interferon-gamma-mediated downregulation of cholesterol efflux and $\mathrm{ABC} 1$ expression is by the Stat 1 pathway. Arteriosclerosis Thrombosis and Vascular Biology. 2002; 22(5):e5-9.

Wågsäter D, Olofsson PS, Norgren L, Stenberg B, Sirsjö A. The chemokine and scavenger receptor CXCL16/SR-PSOX is expressed in human vascular smooth muscle cells and is induced by 
interferon gamma. Biochemical and Biophysical Research Communications. 2004; 325(4):118793. [PubMed: 15555552]

Whitman SC, Ravisankar P, Elam H, Daugherty A. Exogenous interferon-gamma enhances atherosclerosis in apolipoprotein E-/- mice. American Journal of Pathology. 2000; 157:1819-24. [PubMed: 11106554]

Whitman SC, Ravisankar P, Daugherty A. IFN-gamma deficiency exerts gender-specific effects on atherogenesis in apolipoprotein E-/- mice. Journal of Interferon and Cytokine Research. 2002; 22(6):661-70. [PubMed: 12162876]

Wuttge DM, Zhou X, Sheikine Y, Wagsater D, Stemme V, Hedin U, et al. CXCL16/SR-PSOX is an interferon-\{gamma $\}$-regulated chemokine and scavenger receptor expressed in atherosclerotic lesions. Arteriosclerosis Thrombosis and Vascular Biology. 2004; 24(4):750-5.

Zhang J, Alcaide P, Liu L, Sun J, He A, Luscinskas FW, et al. Regulation of endothelial cell adhesion molecule expression by mast cells, macrophages, and neutrophils. PLoS One. 2011; 6(1):e14525. [PubMed: 21264293] 


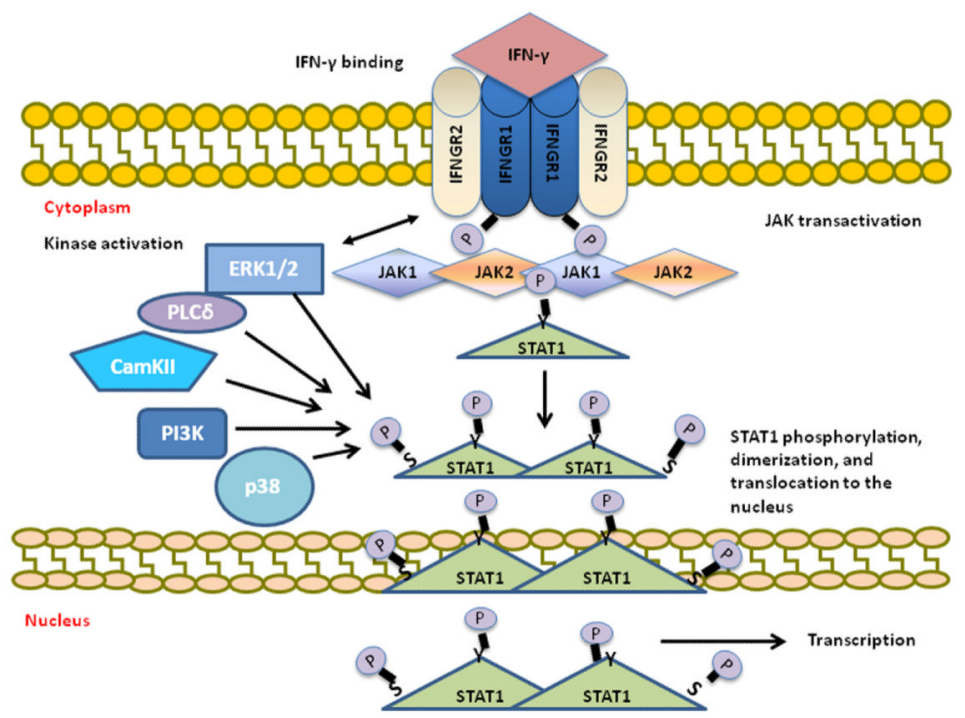

Fig. 1.

A schematic representation of the IFN- $\gamma$ signaling pathway. The receptor for IFN- $\gamma$ consists of two subunits: IFNGR1, the ligand-binding chain and IFNGR2, the signal-transducing chain. Upon interaction of IFN- $\gamma$ with the ligand-binding chain, it dimerizes with the signaltransducing chain. Ligand-induced receptor clustering activates the Janus kinases JAK1 and JAK2 and phosphorylation of a tyrosine residue on the intracellular domain of IFNGR1. Phospho-STAT1 dimerizes, dissociates from the receptor cytoplasmic domain, and translocates to the nucleus to activate a wide range of IFN- $\gamma$-responsive genes. After signaling, the ligand-binding chains are internalized. The chains are then recycled to the cell surface. 


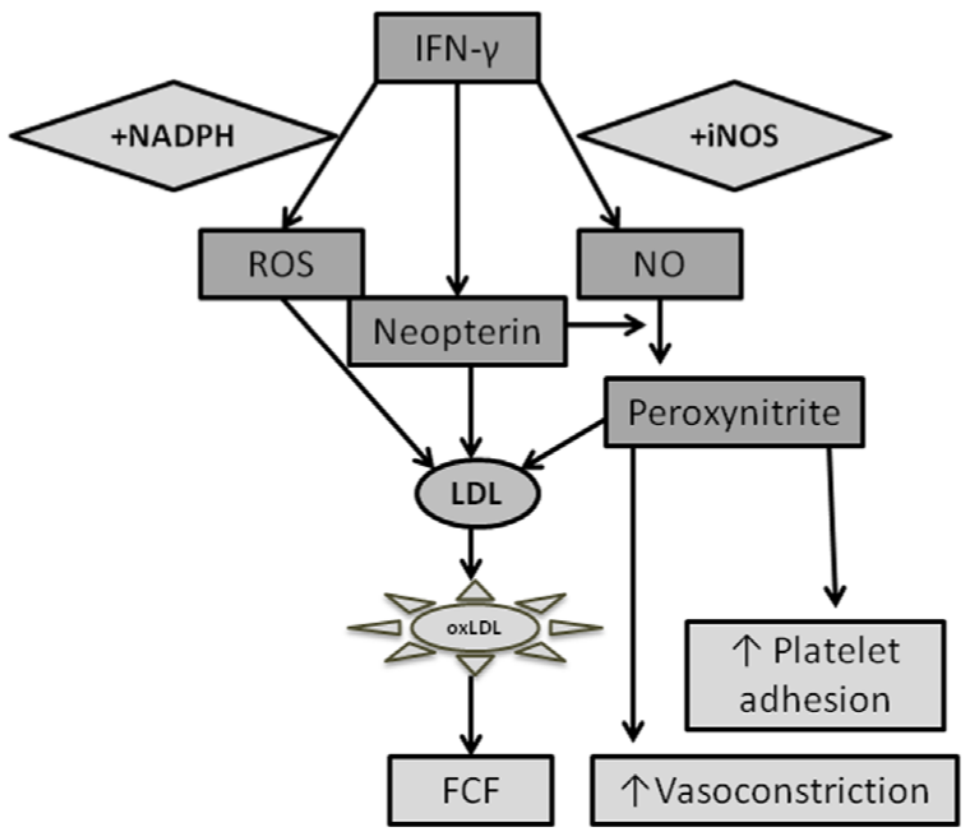

Fig. 2.

The role of IFN- $\gamma$ in oxidative-stress progression and initiation of atherosclerosis. IFN- $\gamma$ is able to upregulate the expression of the superoxide-producing enzyme NADPH oxidase in human monocytes/macrophages, thus inducing ROS production. ROS induces oxidative modification of LDL. Oxidized LDL (oxLDL) is internalized by macrophage scavenger receptors, leading to lipid overload and foam cell formation (FCF). IFN- $\gamma$-induced activation of human macrophages increases the concentration of neopterin. Neopterin may act prooxidatively and supports LDL oxidation in vitro. IFN- $\gamma$ stimulates iNOS-mediated elevation of nitric oxide (NO) concentration. Local release of a large amount of NO metabolites leads to production of peroxynitrite (accelerated by neopterin as well). Accumulation of peroxynitrite enhances platelet adhesion and aggregation, promotes lipid peroxidation, and increases vasoconstriction. 


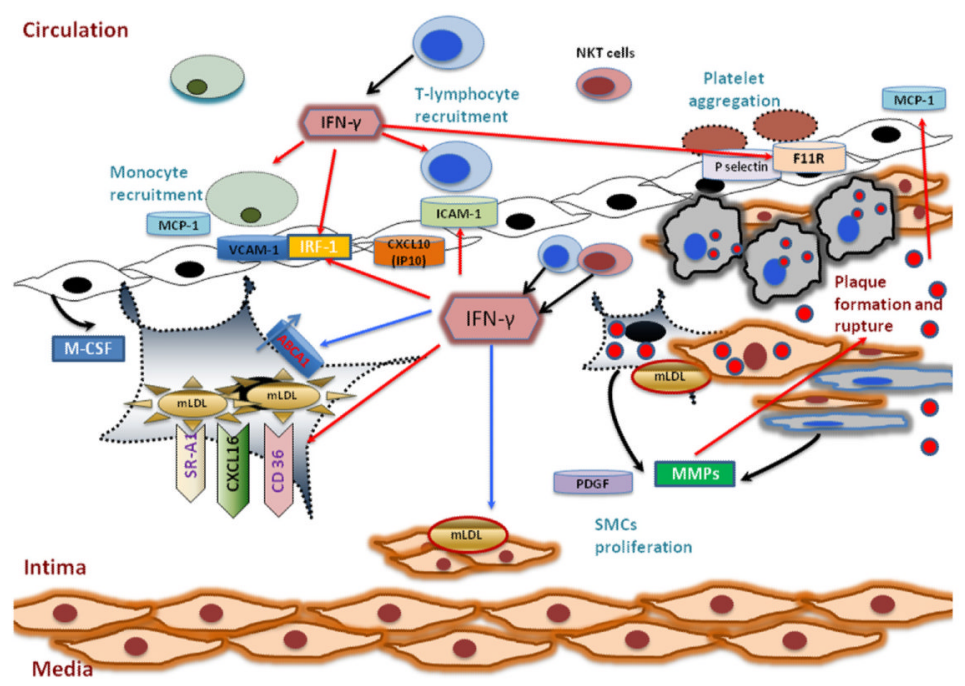

Fig. 3.

A schematic representation of IFN- $\gamma$ effects on atherosclerotic lesion formation. IFN- $\gamma$, secreted by the infiltrating T lymphocytes and natural killer T cells (NKT) $\bullet$, induces (red arrows) or inhibits (blue arrows) the expression of numerous genes in endothelial cells $\Longleftrightarrow$, macrophages $\ominus$, and SMC $\Longleftrightarrow$. IFN- $\gamma$ provokes monocyte/lymphocyte recruitment and infiltration into the subendothelium by induction of MCP-1, M-CSF, and release of adhesion molecules such as ICAM-1, VCAM-1, and P-selectin. IFN- $\gamma$ promotes foam cell formation by increasing the uptake of modified LDL (mLDL $\bullet$ ) by scavenger receptors, like CD36, SRA1, and CXCL16, and reducing cholesterol efflux (ABCA1). IFN$\gamma$-induced release of MMPs enables SMC to migrate through tissue by degrading extracellular matrix. Mature SMC retain remarkable plasticity, become macrophage-like and contribute to the foam cell population of the intima. MMPs are proteolytic enzymes that can weaken and disrupt plaque structure, including the fibrous cap. 


\section{Table 1}

Genes involved in lipid accumulation and foam cell formation regulated by IFN- $\gamma$.

\begin{tabular}{ll}
\hline Gene regulated by IFN- $\gamma$ & References \\
\hline $\begin{array}{l}\text { Downregulated cholesterol efflux } \\
\downarrow \text { ABCA1 }\end{array}$ & Wang et al. (2002), Hao et al. (2009), McLaren and Ramji (2009) \\
$\downarrow$ Liver X receptor & Hao et al. (2009) \\
$\downarrow$ Cholesterol-27-hydroxylase & Reiss et al. (2001), Reiss et al. (2004), McLaren and Ramji (2009) \\
$\begin{array}{l}\text { Augmented cholesterol influx } \\
\uparrow \text { CD36 }\end{array}$ & Tenger et al. (2005), McLaren and Ramji (2009) \\
$\uparrow$ Scavenger receptor A & Grewal et al. (2001), Paulnock et al. (2000), Tenger et al. (2005), McLaren and Ramji (2009) \\
$\uparrow$ CXCL16 & Wagsater et al. (2004), Wuttage et al. (2004), Tenger et al. (2005) \\
\hline
\end{tabular}

